\title{
Polarization-Resolved Extreme-Ultraviolet Second-Harmonic Generation from $\mathrm{LiNbO}_{3}$
}

\author{
Can B. Uzundal, ${ }^{1,2}$ Sasawat Jamnuch $\odot,{ }^{3}$ Emma Berger $\odot,{ }^{1,2}$ Clarisse Woodahl,,${ }^{1,4}$ Paul Manset $\odot,{ }^{5}$ Yasuyuki Hirata, ${ }^{6}$ \\ Toshihide Sumi, ${ }^{7}$ Angelique Amado, ${ }^{1,2}$ Hisazumi Akai $\odot,{ }^{7}$ Yuya Kubota, ${ }^{8,9}$ Shigeki Owada, ${ }^{8,9}$ Kensuke Tono, ${ }^{8,9}$ \\ Makina Yabashi, ${ }^{8,9}$ John W. Freeland,${ }^{10}$ Craig P. Schwartz $\odot,{ }^{11}$ Walter S. Drisdell, ${ }^{12,13}$ Iwao Matsuda $\odot,{ }^{14,7}$ \\ Tod A. Pascal,$^{3,15,16, *}$ Alfred Zong $\oplus^{1,2}$ and Michael Zuerch $\odot^{1,2,17,18, \uparrow}$ \\ ${ }^{1}$ Department of Chemistry, University of California, Berkeley, California 94720, USA \\ ${ }^{2}$ Materials Sciences Division, Lawrence Berkeley National Laboratory, Berkeley, California 94720, USA \\ ${ }^{3}$ ATLAS Materials Science Laboratory, Department of NanoEngineering and Chemical Engineering, University of California, \\ San Diego, La Jolla, California, 92023, USA \\ ${ }^{4}$ University of Florida, Gainesville, Florida 32611, USA \\ ${ }^{5}$ Ecole Normale Superieure de Paris, Paris, France \\ ${ }^{6}$ National Defense Academy of Japan, Yokosuka, Kanagawa 239-8686, Japan \\ ${ }^{7}$ Institute for Solid State Physics, The University of Tokyo, Kashiwa, Chiba 277-8581, Japan \\ ${ }^{8}$ RIKEN SPring-8 Center, 1-1-1 Kouto, Sayo, Hyogo 679-5148, Japan \\ ${ }^{9}$ Japan Synchrotron Radiation Research Institute, (JASRI), 1-1-1 Kouto, Sayo, Hyogo 679-5198, Japan \\ ${ }^{10}$ Advanced Photon Source, Argonne National Laboratory, Argonne, Illinois 60439, USA \\ ${ }^{11}$ Nevada Extreme Conditions Laboratory, University of Nevada, Las Vegas, Las Vegas, Nevada 89154, USA \\ ${ }^{12}$ Chemical Sciences Division, Lawrence Berkeley National Laboratory, Berkeley, California 94720, USA \\ ${ }^{13}$ Joint Center for Artificial Photosynthesis, Lawrence Berkeley National Laboratory, Berkeley, California 94720, USA \\ ${ }^{14}$ Trans-scale Quantum Science Institute, The University of Tokyo, Bunkyo-ku, Tokyo 113-0033, Japan \\ ${ }^{15}$ Materials Science and Engineering, University of California San Diego, La Jolla, California, 92023, USA \\ ${ }^{16}$ Sustainable Power and Energy Center, University of California San Diego, La Jolla, California, 92023, USA \\ ${ }^{17}$ Fritz Haber Institute of the Max Planck Society, 14195 Berlin, Germany \\ ${ }^{18}$ Friedrich Schiller University Jena, 07743 Jena, Germany
}

(Received 2 April 2021; revised 21 August 2021; accepted 15 October 2021; published 30 November 2021)

Second harmonic generation (SHG) spectroscopy ubiquitously enables the investigation of surface chemistry, interfacial chemistry, as well as symmetry properties in solids. Polarization-resolved SHG spectroscopy in the visible to infrared regime is regularly used to investigate electronic and magnetic order through their angular anisotropies within the crystal structure. However, the increasing complexity of novel materials and emerging phenomena hampers the interpretation of experiments solely based on the investigation of hybridized valence states. Here, polarization-resolved SHG in the extreme ultraviolet (XUV-SHG) is demonstrated for the first time, enabling element-resolved angular anisotropy investigations. In noncentrosymmetric $\mathrm{LiNbO}_{3}$, elemental contributions by lithium and niobium are clearly distinguished by energy dependent XUV-SHG measurements. This element-resolved and symmetrysensitive experiment suggests that the displacement of $\mathrm{Li}$ ions in $\mathrm{LiNbO}_{3}$, which is known to lead to ferroelectricity, is accompanied by distortions to the $\mathrm{Nb}$ ion environment that breaks the inversion symmetry of the $\mathrm{NbO}_{6}$ octahedron as well. Our simulations show that the measured second harmonic spectrum is consistent with $\mathrm{Li}$ ion displacements from the centrosymmetric position while the $\mathrm{Nb}-\mathrm{O}$ bonds are elongated and contracted by displacements of the $\mathrm{O}$ atoms. In addition, the polarization-resolved measurement of XUV-SHG shows excellent agreement with numerical predictions based on dipoleinduced SHG commonly used in the optical wavelengths. Our result constitutes the first verification of the dipole-based SHG model in the XUV regime. The findings of this work pave the way for future angle and time-resolved XUV-SHG studies with elemental specificity in condensed matter systems.

DOI: 10.1103/PhysRevLett.127.237402

Published by the American Physical Society under the terms of the Creative Commons Attribution 4.0 International license. Further distribution of this work must maintain attribution to the author(s) and the published article's title, journal citation, and DOI.
Nonlinear spectroscopies have become an indispensable tool to characterize material properties and dynamics. Second-order optical nonlinearities proportional to the second-order susceptibility $\chi^{(2)}$ are especially relevant due to their distinct selection rules beyond the angular momentum selection rule. Factors such as the bulk symmetry of the 
crystal and the presence of an inversion center determine whether second-order susceptibilities are nonzero [1]. Owing to these properties, second-order nonlinear spectroscopies, such as second harmonic generation (SHG) with optical and infrared wavelengths, are widely used as an interfacial and surface probe of electronic properties of solid state materials [2].

Because of the nonlinear process, a high electric field strength is needed to generate experimentally detectable SHG signals. In this regard, advances in pulsed laser sources with a high peak field strength greatly accelerated the adoption of this technique. In the optical regime, besides direct measurement of SHG, the angular anisotropy of SHG is often used to characterize electronic and magnetic orders in solids, offering an ultrasensitive probe of crystalline symmetry [3]. For example, SHG angular anisotropy has been used to characterize the symmetries of ferroic materials [4,5], multipolar order [6], and chiral structures [7,8]. The technique has been used extensively in both transmission and reflection geometry, with the latter first described in detail in 1962 [9]. In systems with strong electron correlations-from unconventional superconductors to quantum spin liquid-the sensitivity afforded by SHG has also revealed important phases that eluded previous investigations [10-12]. Free-electron lasers (FELs) present a similar opportunity to extend the capabilities of SHG into the extreme ultraviolet (XUV) and soft x-ray regime [13] as well as the terahertz region [14]. Recently, nonlinear $\mathrm{x}$-ray and XUV spectroscopies have been the subjects of both theoretical [15] and experimental [16-20] work as a result of the available high intensity light sources. Among these techniques, XUV-SHG is particularly attractive as the corelevel specificity of XUV radiation and the unique selection rules of SHG can be united. Further, short pulse durations pushing to the attosecond regime that are available at the current and upcoming generation of FELs are expected to enable XUV-SHG studies with exceptional time resolution [21]. Experimentally, distinct elemental edges can be probed using XUV-SHG $[13,22]$. Following these proof of principle works, XUV-SHG was also shown to probe material properties with high sensitivity to the chemical environment around the select elements [23] and was demonstrated as a spectroscopic tool capable of probing buried interfaces [24].

In contrast to optical SHG that measures an average response across the elements forming the valence orbitals, XUV-SHG probes the element-specific core level states, allowing the separation of elemental contributions in the measured anisotropies. The elemental specificity of XUVSHG is particularly attractive for materials where emergent behavior is rooted in single ion displacements in the unit cell. For instance, in ferroelectric materials, a spontaneous polarization forms as a result of unit cell distortions that break the inversion symmetry. In particular, for the ferroelectric material studied in this Letter, $\mathrm{LiNbO}_{3}$, the spontaneous polarization establishes as a result of the $\mathrm{Li}$ displacement relative to the $\mathrm{Nb}-\mathrm{O}$ octahedron in the unit cell.

Here, polarization-resolved XUV-SHG is demonstrated for the first time and applied to study the nature of symmetry-breaking ion displacement in ferroelectric $\mathrm{LiNbO}_{3}$. Spectroscopy measurements covering the Li $K$ and $\mathrm{Nb} N$ edges were conducted in concert with polarization-resolved studies at an FEL. Resonant features relating to the two elements are observed and assigned using ab initio density functional perturbation theory (DFPT). This technique was first used in the visible spectrum to understand nonlinear phenomena [25]. Significantly, the method captures the effect of resonance on second harmonic generation. It has been shown that this method can be successfully applied to x-ray transitions [13,23]. Here, the angular anisotropy of a selected resonance is clearly resolved, which is well reproduced by the theory of nonlinear polarization based on DFPT calculations.

The experiments were performed at BL1 of SACLA in Japan [26]. A 30-fs $p$-polarized FEL pulse was tuned to energies between 28 and $33 \mathrm{eV}$ with $0.5 \mathrm{eV}$ steps and incident on an $x$-cut $\mathrm{LiNbO}_{3}$ crystal (Fig. S5 [27]) at $45^{\circ}$ with respect to the sample surface. The incoming photon energies are referred as the fundamental in the remaining text. The second harmonic response of the sample was analyzed in two separate experiments.

In the first experiment, as illustrated in Fig. 1(a), the second harmonic and the reflected fundamental were dispersed by a grating (1200 groove/mm, 30-002, Shimadzu) and captured using a microchannel plate detector (MCP) (Rectangular, Hamamatsu Photonics) coated with CsI. The images of the detector were captured with a camera (IPX-VGA120-LMCN, Imperx Inc.). This measurement was used to retrieve the second order susceptibility spectrum, $\chi_{\mathrm{eff}}^{(2)}(\omega)$. We emphasize that both the fundamental and the second harmonic light were simultaneously recorded on the detector, allowing a comparison of the shot-to-shot variation in the photon flux and energy. The photon energy jitter was approximately $0.2 \%$ of the fundamental frequency and was leveraged to increase the spectral resolution (see Supplemental Material, Sec. S1 [27]). Shot-to-shot fluctuations in the photon flux of the fundamental was used to extract the second-order susceptibility [Fig. S2(b) [27] ].

In the second experiment [Fig. 1(b)], the polarization of the second harmonic was investigated with an XUV polarizer. Specifically, the second harmonic emitted from the sample was reflected off a multilayer mirror at Brewster's angle, such that only the $s$-polarized portion of the incident light was reflected. The reflected light was then detected by an MCP. The multilayer mirror and the MCP were rotated azimuthally around the beam axis $[\Phi$ in Fig. 1(b)] between $-15^{\circ}$ to $115^{\circ}$ in $3^{\circ}$ steps with respect to the optical table. As the multilayer mirror was rotated, the portion of light that is $p$ polarized with respect to the mirror 

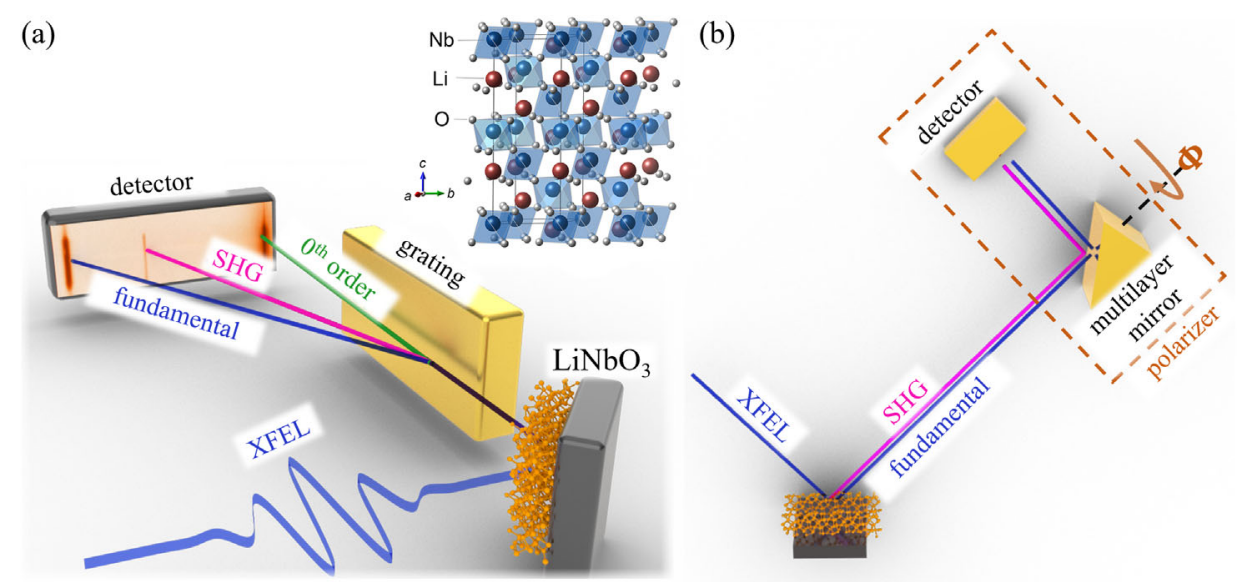

FIG. 1. Schematic overviews of the two experiments. In both experiments the $p$-polarized fundamental is incident on an $x$-cut $\mathrm{LiNbO}_{3}$ sample at $45^{\circ}$ with respect to the crystal surface. The $45^{\circ}$ incident angle is the Brewster's angle for XUV energies. SHG is also detected at $45^{\circ}$ with respect to the crystal surface. (a) Schematic illustration of the spectral measurement. The residual fundamental and the emitted second harmonic of the incident FEL are analyzed by dispersing with a grating and imaging with an MCP detector. The inset shows the crystal structure of $\mathrm{LiNbO}_{3}$ in its ferroelectric state where black lines mark the unit cell. (b) Schematic illustration of the second harmonic polarization measurement. The residual fundamental and the emitted second harmonic are reflected off of a multilayer mirror tuned to $66 \mathrm{eV}$. The polarization of the residual fundamental and the second harmonic are simultaneously measured. Crystal drawing is produced by VESTA [36].

surface with the new orientation is refracted into the mirror substrate and absorbed, while the $s$-polarized component is reflected. The multilayer mirror was coated such that the second harmonic is preferentially reflected over the fundamental, making the separation of the two possible [37]. Nonetheless, a small fraction of fundamental was able to reach the MCP due to nonperfect extinction on the multilayer mirror. Inspecting Fig. S3, one can estimate that the fundamental and second harmonic intensities are approximately the same order of magnitude. As a result of the residual fundamental at the detector, the polarization of the fundamental and the second harmonic can be resolved simultaneously [27].

A detailed analysis procedure for the spectral data is presented in Sec. S1 of the Supplemental Material [27]. Briefly, each FEL shot imprints a 2D image on the MCP detector. Each image contains peaks associated with the incident fundamental and the emitted second harmonic at their respective frequencies. The quality of each shot was determined by fitting a Gaussian to the fundamental peak and assessing the quality of the fit. Approximately $5 \%$ of all shots at each energy were discarded on the basis of $R^{2}$ values less than 0.9 . The remaining shots were binned with respect to the fundamental energy and intensity. The second order susceptibility at each energy was extracted using the quadratic relationship between the fundamental intensity and its second harmonic.

The detector in the polarization-resolved experiment measures both the reflected fundamental and the emitted second harmonic as a function of angle $\Phi$ as shown in Fig. 1(b). A detailed procedure for the data analysis steps for the polarization experiment is presented in the
Supplemental Material, Sec. S2 [27]. Briefly, the fundamental and the second harmonic response were separated by a linear background subtraction. At lower incident fundamental intensities, the measured voltage was linear with respect to the intensity of the incident fundamental while at higher incident fundamental intensities a quadratic relationship is observed.

These experiments were corroborated by theoretical calculations. The linear response of $\mathrm{LiNbO}_{3}$ was simulated with first-principles DFPT [38] using exciting [39] full potential all electron augmented linearized plane wave package. Both centrosymmetric and noncentrosymmetric structures of $\mathrm{LiNbO}_{3}$ were investigated. The Brillouin zone was sampled with a $15 \times 15 \times 15 \Gamma$-point centered $k$-point grid within the local density approximation [40]. The Li core $1 s$ and $\mathrm{Nb}$ semicore $4 s$ and $4 p$ electrons were included in the self-consistent field calculation loop to extract their respective linear responses. The excited states of the system were accessed through time-dependent density functional theory (TDDFT) simulations using the random phase approximation (RPA) kernel implemented within exciting $[41,42]$ with a $q$-point chosen to be the same as that of the aforementioned $k$-point grid. Plots of imaginary part of the dielectric function are shown in Fig. S12 [27]. No characteristic differences to the linear dielectric response were found between the nonpolar and polar phases. The experimental and theoretical results show good agreement except the overestimation of the first peak at $36 \mathrm{eV}$ as shown in Fig. S12 [27], which is a common trait in the level of theory used here. To properly sample the $t_{2 g}$ peak, many-body effects would need to be included, but is beyond the scope of the present work and not necessary for the current level 
of analysis. The level of theory employed in the linear response calculation was kept at the same level as the second harmonic response calculation for consistency and ease of comparison. The second harmonic response formalism by Sharma [25] implemented within exciting [39] was used to calculate the second order susceptibility of $\mathrm{LiNbO}_{3}$. Here, lifetime broadening was also employed to account for the high oscillatory behavior of high-energy states as detailed in the work of Lam et al. [13]. A total of 120 empty states were included to account for excitation up to double of the incoming photon frequency. Molecular dynamics simulations were performed to correctly reflect the finite temperature of the system (see Supplemental Material, Sec. S4 [27] for details).

Considering the electronic density of states, the core level transitions that can be accessed by XUV-SHG is schematically shown in Fig. 2(a). Half resonant transitions from $\mathrm{Li} 1 s$ core states to the conduction band states with majority $\mathrm{Nb} 4 d$ character, along with resonant transitions originating from $\mathrm{Nb} 4 p$ corelike states fall within the range of the fundamental energies studied. Though the availability of density of states is vital for observing XUV-SHG, it is not the only factor that governs the transition probability. Experimentally, the measured second order susceptibility spectrum $\chi_{\mathrm{eff}}^{(2)}$ is a direct measurement of the allowed transitions within the selection rules of XUV-SHG. In Fig. 2(b) the measured $\chi_{\text {eff }}^{(2)}$ is overlaid with the theoretically calculated spectrum for $\mathrm{LiNbO}_{3}$. The error bars are derived from the fluctuation of the signal at a given photon energy value. The FEL pulse energy varies roughly randomly within a nearly Gaussian-shaped envelope. Furthermore, every photon energy setting has a slightly different average pulse energy value depending on how well the FEL could be tuned at a given value. This has two consequences: (i) certain energy values were sampled substantially more frequently than other energy values, and (ii) due to the nonlinear nature of the SHG signal, the signal-to-noise affects the fidelity of the nonlinear fit depending on the average pulse energy. Qualitatively, the higher the average pulse energy at a given photon energy, the better the signal-to-noise for that data point in the SHG spectrum. The combination of both of these effects leads to large differences in experimental errors. The theoretically calculated spectrum is derived as a weighted sum of the individual $\chi^{(2)}$ tensor elements of the $\mathrm{C}_{3 v}$ point group under consideration of the experimental geometry (see Sec. S3 in Ref. [27] for details). Note that the theoretical calculations are not absolutely calibrated in terms of energy scale. The two half-resonant features around $58 \mathrm{eV}$ correspond to transitions from $\mathrm{Li} 1 s$ to conduction band states of majority $\mathrm{Nb} 4 d$ character, assigned based both on theoretical and experimental concerns (Supplemental Material, Sec. S5 [27]). These features report on the ferroelectric displacement in the unit cell involving the Li ions. Our TDDFT calculations (Supplemental Material, Sec. S5 [27]) are consistent with an inversion symmetry breaking displacement of $\mathrm{Li}$ ions. The resonant feature at $58 \mathrm{eV}$ is similar to the feature
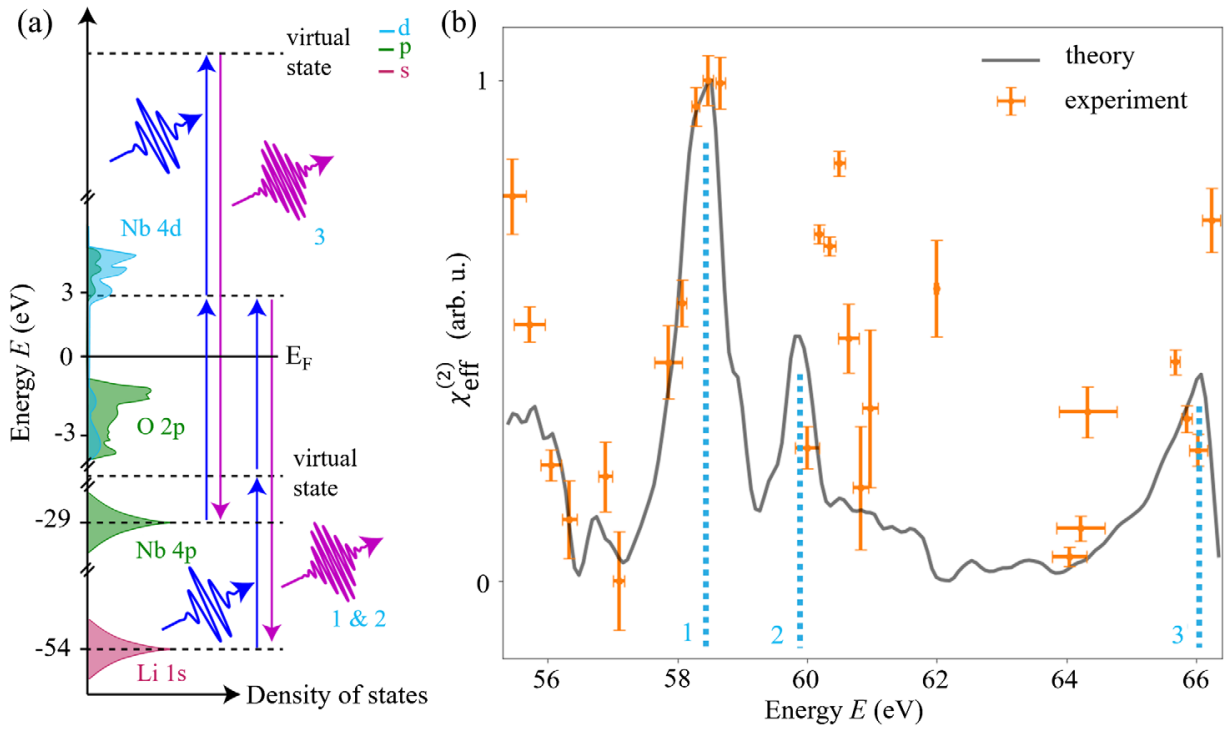

FIG. 2. Resonant transitions for $\mathrm{LiNbO}_{3}$ within the range of fundamental energies studied in the spectral experiment. (a) Schematic illustration of the possible transitions with respect to the electronic density of states. Two prominent transitions that involve the Li $1 s$ and $\mathrm{Nb} 4 p$ core states are labeled. For the Li $1 s$ states (transitions 1 and 2), SHG occurs through a half-resonant scheme and it is facilitated by a virtual state, while the transition originating from $\mathrm{Nb} 4 p$ (transition 3 ) is facilitated via the conduction band states of majority $\mathrm{Nb} 4 d$ character. (b) Measured and theoretical values of the effective $\chi_{\text {eff }}^{(2)}(\omega)$ spectrum for $\mathrm{LiNbO}_{3}$ showing three prominent features corresponding to the labeled transitions from panel (a). 
observed in a previous XUV-SHG study on $\mathrm{LiOsO}_{3}$ [23]. The previous study reported an enhancement of the $\chi^{(2)}$ amplitude with inversion symmetry breaking in the unit cell by Li displacements. In fact, the behavior of the two Li containing compounds with ferroelectric displacements, $\mathrm{LiNbO}_{3}$ and $\mathrm{LiOsO}_{3}$ is very similar in this energy range. The key difference in the $\chi_{\text {eff }}^{(2)}$ spectra is highlighted in Fig. 2(b) as transition No. 3. This highlighted feature in Fig. 2(b) stems from half-resonant transitions from the $\mathrm{Nb} N$ edge, pointing towards inversion symmetry breaking not only around the $\mathrm{Li}$ ion but also the $\mathrm{Nb}$ ion. Molecular dynamics simulations of the $\mathrm{Nb}$ ion environment in the presence of the ferroelectric displacement show that the amplitude of $\chi_{\text {eff }}^{(2)}$ at $2 \hbar \omega=66 \mathrm{eV}$ is consistent with a modulation of the $\mathrm{Nb}-\mathrm{O}$ bonds. The molecular dynamics simulations also show that antiferrodistortive rotations of the $\mathrm{NbO}_{6}$ do not substantially contribute to the amplitude of the $\chi_{\text {eff }}^{(2)}$ spectrum shown in Fig. 2(b). This result is consistent with a picture of ferroelectric displacement of the $\mathrm{Li}$ ion that is stabilized by distortions to the $\mathrm{NbO}_{6}$ octahedra that break the inversion symmetry around the $\mathrm{Nb}$ ion in the unit cell [43-46]. No evidence of such inversion symmetry breaking around the Os ion in $\mathrm{LiOsO}_{3}$ was observed [23]. This contrast between the two cases demonstrates the strength of the unique selection rules of XUV-SHG and is a direct visualization of element specificity of the method. Further improvement in the agreement between theory and experiment can be achieved by going beyond the approximations inherent in TDDFT and linear response theory and more precise measurements with increased statistics.

To further demonstrate the capabilities of energyresolved SHG, we conducted polarization-resolved measurements at a single energy. Along with the fundamental $(\hbar \omega=33 \mathrm{eV})$ that initiates SHG, the polarization of SHG at $2 \hbar \omega=66 \mathrm{eV}$ is resolved and shown as a polar plot in Fig. 3. The accessible angles were limited by what was experimentally feasible at SACLA at the time. The reflected fundamental signal is $p$ polarized while the second harmonic is $s$ polarized, where the polarizations are referenced with respect to the plane of reflection at the sample. It is worth mentioning that though the fundamental light was incident on the sample at Brewster's angle, some residual $p$ polarized fundamental was still reflected due to imperfect alignment.

Using the well-established arguments for the crystal symmetry and a detailed tensor analysis, the polarization of the second harmonic can be calculated and decomposed into four distinct channels characterized by the polarization of the incident fundamental and that of the second harmonic emitted. All four cases are shown in Fig. S8 [27] as a function of in-plane rotation angles of $x$-cut and $z$-cut $\mathrm{LiNbO}_{3}$. The calculated polar plots for the 4 distinct

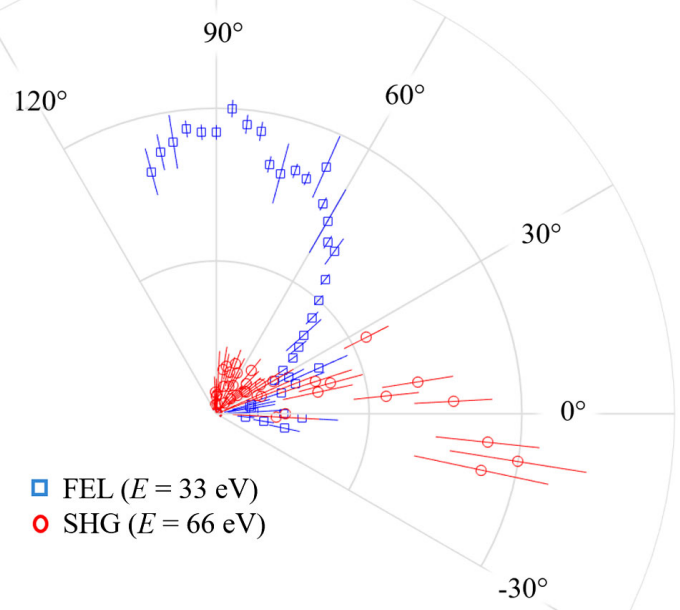

FIG. 3. Results of the polarization-resolved experiment on $x$-cut $\mathrm{LiNbO}_{3}$. Measured polarization of the incident FEL (blue squares) at $33 \mathrm{eV}$ and the polarization of the SHG (red circles). $p$ polarization with respect to the optical table is $90^{\circ}$ while $s$ polarization is $0^{\circ}$. The fitting error is shown for each point. The amplitude of the intensity is in arbitrary units and the scale is linear. The intensities of fundamental and second harmonic are individually normalized by their respective maximum.

channels of polarization ( $p$ in $p$ out, $p$ in $s$ out, $s$ in $p$ out, $s$ in $s$ out) show distinct patterns that are related to the symmetry of the crystal. Note that, as expected, the threefold symmetry of the bulk crystal is reproduced by the polar plots for the $z$-cut crystal. For $x$-cut $\mathrm{LiNbO}_{3}$, when it is incident with a $p$-polarized fundamental, the majority of the emitted second harmonic is calculated to be $s$ polarized [Figs. S7(b) and S7(c) [27]] at $2 \hbar \omega=66 \mathrm{eV}$, in excellent agreement with the measured polarization of the second harmonic. The emitted s-polarized SHG constitutes the emission of the orthogonal polarization to the polarization of the fundamental. The emission of the orthogonal polarization can be attributed to the energy dependent $\chi^{(2)}$ tensor as the emission of $p$-polarized SHG is indeed symmetry allowed as shown in Figs. S7(b) and S7(c) for a different XUV energy. The observed s-polarized SHG thus cannot be solely explained by symmetry considerations and highlights the nonlinear nature of the lightmatter interaction in this regime. While the data taken here are limited to a single crystal angle, as higher repetition rate FELs become available, all of the data taken here can be obtained in a fraction of the time.

The findings presented here demonstrate the feasibility of an XUV-SHG study with angular resolution and the possibility to extend SHG rotational anisotropy studies into XUV wavelengths. The long established theory of nonlinear polarization up to the dipole contributions is shown 
to be adequate to explain the measured polarization for a bulk noncentrosymmetric material in the extreme ultraviolet regime. Our spectral findings suggest that inversion symmetry breaking in the ferroelectric phase of $\mathrm{LiNbO}_{3}$ may not be limited to $\mathrm{Li}$ ion displacements. Contributions to the $\chi_{\text {eff }}^{(2)}$ spectrum from $\mathrm{Nb}$ ions suggest inversion symmetry breaking around the $\mathrm{Nb}$ ions is important as well. There have been recent demonstrations of polarization switching of ferroelectric materials by light [47-49]. We envision that the demonstrated principles can be fully extended to time-resolved studies that leverage the excellent time resolution afforded by the short XUV pulses, paving the way towards attosecond nonlinear spectroscopies on ferroelectrics, surfaces, or buried interfaces with element specificity.

M.Z., C.S., and A. A. acknowledge support by the Max Planck Society (Max Planck Research Group). M. Z. acknowledges support by the Federal Ministry of Education and Research (BMBF) under "Make our Planet Great Again-German Research Initiative" (Grant No. 57427209 "QUESTforENERGY") implemented by DAAD. J. W. F. acknowledges Department of Energy Grant No. DE SC-0012375. W. D. acknowledges support from the Joint Center for Artificial Photosynthesis, a DOE Energy Innovation Hub, supported through the Office of Science of the U.S. Department of Energy, under Award No. DE-SC0004993. A.Z. acknowledges support from the Miller Institute for Basic Research in Science. Measurements were performed at BL1 of SACLA with the approval of the Japan Synchrotron Radiation Research Institute (JASRI) (Proposal No. 2019B8066). This work was supported by the SACLA Basic Development Program 2018-2020. The authors would like to acknowledge the supporting members of the SACLA facility. Additional measurements were performed at beam line 6.3.2 of the Advanced Light Source, a U.S. DOE Office of Science User Facility under Contract No. DE-AC02-05CH11231. This research used resources of the National Energy Research Scientific Computing Center, a DOE Office of Science User Facility supported by the Office of Science of the U.S. Department of Energy under Contract No. DEAC02-05CH11231. This work also used the Extreme Science and Engineering Discovery Environment (XSEDE), which is supported by National Science Foundation Grant No. ACI-1548562. C. W. acknowledges support by the National Science Foundation REU Program Grant No. 1852537. M. Z. acknowledges funding by the W. M. Keck Foundation, funding from the UC Office of the President within the Multicampus Research Programs and Initiatives (M21PL3263), and funding from Laboratory Directed Research and Development Program at Berkeley Lab (107573). We acknowledge Shukai Yu for providing the sample for this study. C. U. is grateful for discussions with Yue Sun.
* Corresponding author. tpascal@ucsd.edu Corresponding author. mwz@berkeley.edu

[1] R. W. Boyd, Nonlinear Optics, 3rd ed. (Academic Press, New York, 2008).

[2] T. F. Heinz, M. M. T. Loy, and W. A. Thompson, Study of Si (111) Surfaces by Optical Second-Harmonic Generation: Reconstruction and Surface Phase Transformation, Phys. Rev. Lett. 54, 63 (1985).

[3] D. H. Torchinsky and D. Hsieh, Rotational Anisotropy Nonlinear Harmonic Generation (Springer, New York, 2017).

[4] S. Denev, A. Kumar, M. D. Biegalski, H. W. Jang, C. M. Folkman, A. Vasudevarao, Y. Han, I. M. Reaney, S. Trolier-McKinstry, C. B. Eom, D. G. Schlom, and V. Gopalan, Magnetic Color Symmetry of Lattice Rotations in a Diamagnetic Material, Phys. Rev. Lett. 100, 257601 (2008).

[5] H. Padmanabhan, Y. Park, D. Puggioni, Y. Yuan, Y. Cao, L. Gasparov, Y. Shi, J. Chakhalian, J. M. Rondinelli, and V. Gopalan, Linear and nonlinear optical probe of the ferroelectric-like phase transition in a polar metal, $\mathrm{LiOsO}_{3}, \mathrm{Appl}$. Phys. Lett. 113, 122906 (2018).

[6] W. Jin, E. Drueke, S. Li, A. Admasu, R. Owen, M. Day, K. Sun, S. Cheong, and L. Zhao, Observation of a ferrorotational order coupled with second-order nonlinear optical fields, Nat. Phys. 16, 42 (2020).

[7] M. Fiebig, V. V. Pavlov, and R. V. Pisarev, Second-harmonic generation as a tool for studying electronic and magnetic structures of crystals: review, J. Opt. Soc. Am. B 22, 96 (2005).

[8] B. T. Fichera, A. Kogar, L. Ye, B. Gökce, A. Zong, J. G. Checkelsky, and N. Gedik, Second harmonic generation as a probe of broken mirror symmetry, Phys. Rev. B 101, 241106 (R) (2020).

[9] N. Bloembergen and P. S. Pershan, Light waves at the boundary of nonlinear media, Phys. Rev. 128, 606 (1962).

[10] J. W. Harter, Z. Y. Zhao, J.-Q. Yan, D. G. Mandrus, and D. Hsieh, A parity-breaking electronic nematic phase transition in the spin-orbit coupled metal $\mathrm{Cd}_{2} \mathrm{Re}_{2} \mathrm{O}_{7}$, Science 356, 295 (2017).

[11] L. Zhao, C. A. Belvin, R. Liang, D. A. Bonn, W. N. Hardy, N. P. Armitage, and D. Hsieh, A global inversion-symmetrybroken phase inside the pseudogap region of $\mathrm{YBa}_{2} \mathrm{Cu}_{3} \mathrm{O}_{y}$, Nat. Phys. 13, 250 (2017).

[12] N. J. Laurita, A. Ron, J. W. Han, A. Scheie, J. P. Sheckelton, R. W. Smaha, W. He, J. J. Wen, J. S. Lee, Y. S. Lee, M. R. Norman, and D. Hsieh, Evidence for a parity broken monoclinic ground state in the $S=1 / 2$ Kagomé antiferromagnet herbertsmithite, arXiv:1910.13606.

[13] R. K. Lam, S. L. Raj, T. A. Pascal, C. D. Pemmaraju, L. Foglia, A. Simoncig, N. Fabris, P. Miotti, C. J. Hull, A. M. Rizzuto et al., Soft X-Ray Second Harmonic Generation as an Interfacial Probe, Phys. Rev. Lett. 120, 023901 (2018).

[14] T. Dekorsy, V. A. Yakovlev, W. Seidel, M. Helm, and F. Keilmann, Infrared-Phonon-Polariton Resonance of the Nonlinear Susceptibility in GaAs, Phys. Rev. Lett. 90, 055508 (2003). 
[15] E. Minerbi and S. Shwartz, Difference frequency generation of ultraviolet from x-ray pulses in opaque materials, J. Opt. Soc. Am. B 36, 624 (2019).

[16] T. E. Glover, D. M. Fritz, M. Cammarata, T. K. Allison, S. Coh, J. M. Feldkamp, H. Lemke, D. Zhu, Y. Feng, R. N. Coffee, M. Fuchs, S. Ghimire, J. Chen, S. Shwartz, D. A. Reis, S.E. Harris, and J. B. Hastings, X-ray and optical wave mixing, Nature (London) 488, 603 (2012).

[17] J. Szlachetko, J. Hoszowska, J. Dousse, M. Nachtegaal, W. Błachucki, Y. Kayser, J. Sà, M. Messerschmidt, S. Boutet, G. J. Williams et al., Establishing nonlinearity thresholds with ultraintense X-ray pulses, Sci. Rep. 6, 33292 (2016).

[18] M. Beye, R. Y. Engel, J. O. Schunck, S. Dziarzhytski, G. Brenner, and P. S. Miedema, Non-linear soft X-ray methods on solids with MUSIX-the multi-dimensional spectroscopy and inelastic x-ray scattering endstation, J. Condens. Matter Phys. 31, 014003 (2019).

[19] R. Bohinc, G. Pamfilidis, J. Rehault, P. Radi, C. Milne, J. Szlachetko, F. Bencivenga, F. Capotondi, R. Cucini, L. Foglia et al., Nonlinear XUV-optical transient grating spectroscopy at the Si $\mathrm{L}_{2,3}$-edge, Appl. Phys. Lett. 114, 181101 (2019).

[20] S. Shwartz, M. Fuchs, J. B. Hastings, Y. Inubushi, T. Ishikawa, T. Katayama, D. A. Reis, T. Sato, K. Tono, M. Yabashi et al., X-Ray Second Harmonic Generation, Phys. Rev. Lett. 112, 163901 (2014).

[21] J. Duris, S. Li, T. Driver, E. G. Champenois, J. P. MacArthur, A. A. Lutman, Z. Zhang, P. Rosenberger, J.W. Aldrich, R. Coffee, G. Coslovich et al., Tunable isolated attosecond $\mathrm{X}$-ray pulses with gigawatt peak power from a free-electron laser, Nat. Photonics 14, 30 (2020).

[22] S. Yamamoto, T. Omi, H. Akai, Y. Kubota, Y. Takahashi, Y. Suzuki, Y. Hirata, K. Yamamoto, R. Yukawa, K. Horiba et al., Element Selectivity in Second-Harmonic Generation of $\mathrm{GaFeO}_{3}$ by a Soft-X-Ray Free-Electron Laser, Phys. Rev. Lett. 120, 223902 (2018).

[23] E. Berger et al., Extreme ultraviolet second harmonic generation spectroscopy in a polar metal, Nano Lett. 21, 6095 (2021).

[24] C. P. Schwartz et al., Angstrom-Resolved Interfacial Structure in Buried Organic-Inorganic Junctions, Phys. Rev. Lett. 127, 096801 (2021).

[25] S. Sharma and C. Ambrosch-Draxl, Second-harmonic optical response from first principles, Phys. Scr. T T109, 128 (2004).

[26] S. Owada, K. Togawa, T. Inagaki, T. Hara, T. Tanaka, Y. Joti, T. Koyama, K. Nakajima, H. Ohashi, Y. Senba et al., A soft X-ray free-electron laser beamline at SACLA: The light source, photon beamline and experimental station, J. Synchrotron Radiat. 25, 282 (2018).

[27] See Supplemental Material at http://link.aps.org/ supplemental/10.1103/PhysRevLett.127.237402 containing additional details of experimental and numerical analysis and methods, which includes Refs. [1,8,28-35].

[28] J. D. Hunter, Matplotlib: A 2D graphics environment, Comput. Sci. Eng. 9, 90 (2007).

[29] C. R. Harris, K. J. Millman, S. J. van der Walt, R. Gommers, P. Virtanen, D. Cournapeau, E. Wieser, J. Taylor, S. Berg,
N. J. Smith et al., Array programming with NumPy, Nature (London) 585, 357 (2020).

[30] P. Virtanen, R. Gommers, T. E. Oliphant, M. Haberland, T. Reddy, D. Cournapeau, E. Burovski, P. Peterson, W. Weckesser, J. Bright et al., SciPy 1.0: Fundamental Algorithms for Scientific Computing in PYTHON, Nat. Methods 17, 261 (2020).

[31] B. Fichera, ShgPy, https://bfichera.github.io/shgpy/ (2020).

[32] C. J. Kaplan, P. M. Kraus, E. M. Gullikson, L. J. Borja, S. K. Cushing, M. Zürch, Hu. Chang, D. M. Neumark, and S. R. Leone, Retrieval of the complex-valued refractive index of germanium near the $\mathrm{M}_{4,5}$ absorption edge, J. Opt. Soc. Am. B 36, 1716 (2019).

[33] L. D. Menard, Q. Wang, J. H. Kang, A. J. Sealey, G. S. Girolami, X. Teng, A. I. Frenkel, and R. G. Nuzzo, Structural characterization of bimetallic nanomaterials with overlapping x-ray absorption edges, Phys. Rev. B 80, 064111 (2009).

[34] J. C. Fuggle and N. Mårtensson, Core-level binding energies in metals, J. Electron Spectrosc. Relat. Phenom. 21, 275 (1980).

[35] J. H. Underwood, E. M. Gullikson, M. Koike, P. J. Batson, P.E. Denham, K. D. Franck, R.E. Tackaberry, and W. F. Steele, Calibration and standards beamline 6.3.2 at the Advanced Light Source, Rev. Sci. Instrum. 67, 3372 (2019).

[36] K. Momma and F. Izumi, Vesta 3 for three-dimensional visualization of crystal, volumetric and morphology data, J. Appl. Crystallogr. 44, 1272 (2011).

[37] D. Attwood, Soft X-Rays and Extreme Ultraviolet Radiation: Principles and Applications (Cambridge University Press, Cambridge, England, 1999).

[38] P. Hohenberg and W. Kohn, Inhomogeneous electron gas, Phys. Rev. 136, B864 (1964).

[39] A. Gulans, S. Kontur, C. Meisenbichler, D. Nabok, P. Pavone, S. Rigamonti, S. Sagmeister, U. Werner, and C. Draxl, exciting: A full-potential all-electron package implementing density-functional theory and many-body perturbation theory, J. Condens. Matter Phys. 26, 363202 (2014).

[40] J. P. Perdew and A. Zunger, Self-interaction correction to density-functional approximations for many-electron systems, Phys. Rev. B 23, 5048 (1981).

[41] N. Vast, L. Reining, V. Olevano, P. Schattschneider, and B. Jouffrey, Local Field Effects in the Electron Energy Loss Spectra of Rutile $\mathrm{TiO}_{2}$, Phys. Rev. Lett. 88, 037601 (2002).

[42] S. Sagmeister and C. Ambrosch-Draxl, Time-dependent density functional theory versus Bethe-Salpeter equation: An all-electron study, Phys. Chem. Chem. Phys. 11, 4451 (2009).

[43] K. Toyoura, M. Ohta, A. Nakamura, and K. Matsunaga, First-principles study on phase transition and ferroelectricity in lithium niobate and tantalate, J. Appl. Phys. 118, 064103 (2015).

[44] A. S. Barker and R. Loudon, Dielectric properties and optical phonons in $\mathrm{LiNbO}_{3}$, Phys. Rev. 158, 433 (1967).

[45] I. Inbar and R.E. Cohen, Comparison of the electronic structures and energetics of ferroelectric $\mathrm{LiNbO}_{3}$ and $\mathrm{LiTaO}_{3}$, Phys. Rev. B 53, 1193 (1996). 
[46] I. Inbar and R. E. Cohen, Origin of ferroelectricity in $\mathrm{LiNbO}_{3}$ and $\mathrm{LiTaO}_{3}$, Ferroelectrics 194, 83 (1997).

[47] R. Mankowsky, A. von Hoegen, M. Först, and A. Cavalleri, Ultrafast Reversal of the Ferroelectric Polarization, Phys. Rev. Lett. 118, 197601 (2017).

[48] T. Qi, Y.-H. Shin, K.-L. Yeh, K. A. Nelson, and A. M. Rappe, Collective Coherent Control: Synchronization of
Polarization in Ferroelectric $\mathrm{PbTiO}_{3}$ by Shaped THz Fields, Phys. Rev. Lett. 102, 247603 (2009).

[49] E. Mishina, K. Grishunin, V. Bilyk, N. Sherstyuk, A. Sigov, V. Mukhortov, A. Ovchinnikov, and A. Kimel, Polarization switching in ferroelectric thin film induced by a single-period terahertz pulse, MRS Adv. 3, 1901 (2018). 